\title{
A Pesquisa Qualitativa e o Campo da Hospitalidade: uma reflexão sobre a relação teoria e método
}

Maria do Rosário Rolfsen Salles

\begin{abstract}
Resumo
0 artigo procura discutir a relação entre a pesquisa qualitativa e o campo teórico da hospitalidade, com o objetivo de discutir a contribuição dos estudos sobre a hospitalidade em suas diferentes dimensões, para a pesquisa social, tendo em vista o recurso a metodologías qualitativas. Para tanto, recorreu-se à bibliografía sobre o assunto, estabelecendo um recorte na trajetória e evolução histórica da pesquisa qualitativa, procurando establecer relações com os estudos no campo da hospitalidade. Os resultados indicam que a pesquisa no campo da hospitalidade, na sua dupla dimensão, como fato social e como valor, tem se valido da evolução e diversificação de metodologías qualitativas que evidenciam a enorme relação entre ambas, pesquisa qualitativa e hospitalidade.
\end{abstract}

Palavras-chave: Hospitalidade; Pesquisa social; Metodologias qualitativas.

\section{Abstract \\ Qualitative research and the field of hospitality: a reflection on the relationship between theory and method}

The article discusses the relationship between qualitative research and the theoretical field of hospitality, in order to discuss the contribution of studies on hospitality in its various dimensions, for social research, in view of the use of qualitative methodologies. Therefore, we used the bibliography on the subject, establishing a cutout in the background and historical development of qualitative research, looking for relations with the studies in the field of hospitality. The results indicate that research in the field of hospitality, in its dual role as a social phenomenon and as a value, has taken advantage of the evolution and diversification of qualitative methodologies that show the intense relation between both, qualitative research and hospitality.

Keywords: Hospitality; Social research; Qualitative methodologies.

\section{Resumen}

La investigación cualitativa y el campo de la hospitalidad: una reflexión sobre la relación entre la teoría y el método

El articulo analiza la relación entre la investigación cualitativa y el campo teórico de la hospitalidad, con el fin de discutir la contribución de los estudios en la hospitalidad en sus distintas dimensiones, para la investigación social, en vista de la utilización de metodologías cualitativas. Por lo tanto, hemos utilizado la bibliografía sobre el tema, se establece un recorte en el fondo y el desarrollo histórico de la investigación cualitativa, buscando establecer relaciones con los estudios en el campo de la hospitalidad. Los resultados indican que la investigación en el campo de la hospitalidad, en su doble papel

a. Docente e pesquisadora junto ao Programa de Mestrado em Hospitalidade da Universidade Anhembi Morumbi, São Paulo, São Paulo, Brasil. E-mail.mrrsalles@uol.com.br 
como hecho social e como valor, se ha aprovechado de la evolución y diversificación de las metodologías cualitativas que muestran el gran partido entre tanto la investigación cualitativa y hospitalidad.

Palabras clave: Hospitalidad; Investigación social; Metodologías cualitativas.

\section{INTRODUÇÃO}

A investigação científica dos fenômenos sociais, e particularmente da hospitalidade, nos termos em que se configura no presente, é atividade relativamente recente. Desta forma, é preciso delimitar o campo da realidade a que corresponde a hospitalidade dentro daqueles já consolidados das ciências sociais, especialmente das ciências sociais aplicadas.

A hospitalidade, particularmente, entendida como uma maneira de se viver em conjunto, regida por regras, ritos e leis, pode ser "[...] concebida não apenas como uma forma essencial da interação social, [...] pode surgir também como uma forma própria de hominização, ou no mínimo, uma das formas mais essenciais da socialização" (MONTANDON, 2003, p.132) e, nesse sentido, entende-se que, a mais ou menos recente "adoção do campo da hospitalidade, não apenas como designação, mas sobretudo como perspectiva teórica, [traz] ganhos de ordem variada para todos os campos correlatos de aplicação da ciência...." (CAMARG0, 2003, p. 7), ou, mais amplamente, para o enriquecimento da discussão dos principais problemas da sociedade contemporânea no âmbito das demais disciplinas afins, visando, como diz o autor, à adoção de uma nova perspectiva de análise para fenômenos já estudados, não apenas a hotelaria e a restauração.

\section{Proposta do trabalho e método}

Em artigo de 2010, que visava discutir os desafios da pesquisa em hospitalidade, levando-se em conta os avanços da pesquisa em torno do tema nas universidades brasileiras que a ele têm se dedicado ${ }^{1}$, SALLES, BUENO e BASTOS, iniciaram uma discussão sobre aspectos teóricos e de pesquisa envolvendo os estudos sobre hospitalidade, a partir do conceito de dádiva desenvolvido por Marcel Mauss, em trabalho originalmente publicado em 1923/24 com versões para o português, particularmente as edições de 1974 e 2001, do Ensaio sobre a dádiva. 0 objetivo era mostrar a contribuição do conceito para a construção do campo teórico da hospitalidade e a decorrente contribuição da interdisciplinaridade para sua constituição, iniciando pela evolução do conceito na história e as possibilidades abertas para a compreensão dos fenômenos sociais.

No presente trabalho, o objetivo é, a partir do debate sobre a cientificidade nas ciências sociais, e do desdobramento das discussões sobre o conceito de

1. Universidade Anhembi Morumbi, Universidade Caxias do Sul, Universidade do Vale do Itajaí e mesmo a Universidade Federal de Pernambuco, de uma perspectiva mais dedicada ao tema da dádiva como fundamento das relações sociais. 
hospitalidade e da compreensão das diferentes dimensões de que se revestem, entender as contribuições dos estudos sobre a hospitalidade para a pesquisa social na utilização de diferentes metodologias de estudo, considerando-se que a hospitalidade, assim como as pesquisas e métodos qualitativos, são ligados aos estudos de fenômenos e fatos humanos que de facto, não são passiveis de mensuração, o que não os torna menos "científicos" ou opostos aos estudos que se utilizam da quantificação. Ao contrario, a especificidade do campo da hospitalidade dentro da evolução da pesquisa qualitativa em Ciências humanas e sociais, tem contribuído para o avanço da discussão dos problemas sociais contemporâneos.

Sem pretender esgotar a produção, o propósito da discussão é refletir sobre a contribuição do campo da hospitalidade para as pesquisas de caráter qualitativo. Nesse sentido, trata-se de um levantamento bibliográfico, embora não exaustivo, sobre a evolução do debate sobre a cientificidade das ciências sociais e as contribuições do campo da hospitalidade à pesquisa social.

\section{O CAMPO E A PESQUISA EM hOSPITALIDADE}

Utilizando-se da reflexão de CALHOUN, (2014), sobre o papel das Ciências Sociais em um mundo em mudança acelerada, CAMARGO, (2015), ressalta que, dentre as mudanças com as quais se defrontam as ciências sociais frente à nova realidade social, a globalização e o individualismo, são as duas lógicas que, em conjunto, balizam o espaço no interior do qual a pesquisa cada vez mais, é chamada a se mover. Entretanto, diz Camargo, (2015, p. 44), "a hospitalidade caminha em direção diferente. Interessam-lhe a proximidade e o encontro, e este é talvez, o seu principal significado, face às lógicas da globalização e do individualismo". Nessa direção, GOTMAN, (1997), igualmente, entende a hospitalidade como uma forma de indivíduos, grupos e famílias se alojarem, se socializarem e usufruírem serviços mútua e reciprocamente, constituindo, reconhecidamente, uma realidade ao mesmo tempo atual e muito antiga. As diversas formas históricas em que se realiza como relação social, supõem uma dimensão coletiva, em que aparece com o caráter de obrigação, associada num determinado momento, à religião e à caridade, mas que hoje se manifestam basicamente em serviços públicos e de proteção social, e no domínio comercial, nos hotéis. Sendo assim, a hospitalidade no mundo atual, ocorre, como dizem os autores, (GOTMAN, 1997 e CAMARGO, 2015), nos interstícios de um cotidiano marcado pela inospitalidade, quando não pela hostilidade.

A pesquisa no campo da hospitalidade assim, na sua dupla dimensão, como fato social e como valor, (CAMARGO, 2015, p. 45), abre espaço para a discussão, no mundo atual, em que prevalece o esgarçamento do tecido social, das contradições e conflitos inerentes à sociedade capitalista. 0 entendimento dos espaços e domínios (doméstico, social e comercial) que compõem os estudos sobre a hospitalidade, conduzem à melhor compreensão das possibilidades e limites da constituição dos vínculos e relações sociais. A hospitalidade assim, "é mais do que um campo de estudo previamente delimitado, como ocorre nas ciências puras e aplicadas" (CAMARGO, 2015, p. 48). Apresenta-se como um novo paradigma para o estudo do relacionamento humano, uma nova perspectiva, um novo olhar. (CAMARGO, 2015, p. 48. CAILLÉ, 1998 ). 
A retomada das contribuições de Marcel Mauss, e das considerações sobre o papel do dom, nas relações sociais, pelo grupo francês M.A.U.S.S (Mouvement anti utilitariste en Sciences Sociales) data de 1981, apoiado em importantes escolas de pensamento além do próprio Mauss, Karl Polianyi, Geog Simmel entre outros, com o objetivo de valorizar uma critica teórica a partir de algumas categorias sociais como a do dom, da democracia, do associativismo, da participação, da economia solidária, da solidariedade etc., (MARTINS, 2007). Segundo o mesmo autor, uma das contribuições centrais de Mauss foi demonstrar que o valor das coisas não pode ser superior ao valor da relação e que o simbolismo é fundamental para a vida social (MARTINS, 2005, p.46). Ou seja, que a lógica mercantil moderna, não substitui as antigas formas de constituição dos vínculos e alianças dentro das sociedades modernas, constituindo-se mesmo um fato social total, "no sentido de que a sociedade inclui todos os fenômenos humanos de natureza econômica, cultural, política e religiosa, [...] sem haver nenhuma hierarquia prévia que justifique uma economia natural, que precederia os demais fenômenos sociais" (MARTINS, 2005, p. 46) supondo, sobretudo, que a natureza dos bens produzidos não é apenas material, mas reveste-se de um caráter simbólico, que sedimenta as relações sociais, o que está na origem do surgimento da obrigação moral coletiva de que se revestem as relações. Esse movimento assim, que explica o social e a constituição mesmo da sociedade, caracteriza-se pelo anti-utilitarismo, que dá nome ao movimento que propõe um novo paradigma para a compreensão dos fenômenos sociais e das relações sociais.

Desta forma, a dádiva (ou dom), compreendida muito mais como recurso paradigmático do que como uma teoria, e a hospitalidade como dimensão da dádiva, são fundamentais para a compreensão da formação dos vínculos sociais. De fato, "[...] podemos pensar as praticas sociais em termos de mediação da alteridade, pela construção de vínculos construídos a partir da circularidade do dar-receber-retribuir, estabelecendo pontes que criam, ampliam ou rompem alianças e vínculos sociais". (BUENO, 2008, p. 10). Como dimensão da ética, a hospitalidade evidencia "a necessidade de criar e alimentar lugares de hospitalidade onde surge a consciência de um destino comum e o sentido de responsabilidade que motiva a ação solidária" (BUENO, citando BAPTISTA, 2002, p. 158).

Por outro lado, no domínio comercial propriamente dito, diz Gotman, (2008, p. 115), "a hospitalidade entrou de tal maneira na linguagem usual da indústria turística, que acabou se tornando sinônimo desta". Além disso, diz a autora, a troca gratuita da doação (dádiva), se sustenta numa equivalência entre a doação e a contra- doação que se reproduzem infinitamente, diferentemente da troca mercantil que supõe que se esgote no simples pagamento da hospitalidade. Essa é uma discussão que se estende pelas abordagens anglo-saxãs por um lado, e a francesa, por outro, alimentando a ideia de que as relações comerciais supõem uma hospitalidade paga e que portanto se esgota no momento em que há o pagamento. Autores como Lashley e Morrison (2004), Lugosi (2008), entre outros, trabalham essa discussão de maneira a evidenciar as interelações entre as diversas dimensões, a social ou cultural, a privada e a comercial, as relações de hospitalidade podendo acontecer na sobreposição e encontro das três dimensões.

Entretanto, com a preocupação de ampliar o alcance do termo para além do domínio comercial e aprimorar as relações em âmbito comercial, LASHLEY (2015), lembra que até o final do século XX, hotéis, restaurantes e bares costu- 
mavam ser sinônimos de oferta de acomodação, alimentação e bebidas, e assim era comum entender locais como cafés, cantinas, lanchonetes, etc, como locais de provisão de hospitalidade e como que se reduzia a hospitalidade à função de receber e ser recebido em âmbito comercial. Entretanto, diz o autor, talvez como consequência involuntária, essa noção de hospitalidade abriu espaço para o estudo do relacionamento entre hóspedes e anfitriões em todos os domínios: privado, cultural, comercial, possibilitando que a hospitalidade passe a ser entendida como característica fundamental e onipresente na vida humana, propondo, então, a noção de hospitabilidade como o entendimento da disposição das pessoas de serem genuinamente hospitaleiras, sem qualquer expectativa de recompensa ou reciprocidade. Entende-se assim, essa colocação, como um avanço no entendimento das dimensões sociais da hospitalidade.

De fato, sem entrar no mérito das diferenças entre as correntes de pensamento sobre a hospitalidade que têm caracterizado as chamadas linhas ou escolas francesa e anglo-saxônica, essa perspectiva, reforçada pelas colocações de LUGOSI (2008), sobre a importância do estudo da dimensão social para a compreensão daquela estritamente comercial, enriqueceram o debate sobre as possibilidades do campo de estudo da hospitalidade. "O espaço criado e as atitudes hospitaleiras e não apenas o serviço oferecido em si, são a marca da virtude do anfitrião comercial hospitaleiro" (CAMARGO, 2015, p. 54).

\section{A hospitalidade no panorama da pesquisa social e A PESQUISA QUALITATIVA}

Do ponto de vista da complexidade das novas abordagens teóricas que tomaram conta da cena teórica e pratica das ciências sociais, e que emergiram nas décadas finais do século XX, muitas são derivadas da complementação com as áreas da semiótica e hermenêutica ${ }^{2}$. As interpretações pós- positivistas e as interpretações derivadas dos próprios atores sociais, são valorizadas, o que leva os pesquisadores a uma tentativa de validação interna e externa aos modelos interacionistas e construtivistas de pesquisa. (ANADÓN, 2006, p. 10).

Em consequência, as estratégias de pesquisa se multiplicam, a teoria enraizada (grounded theory), os estudos de caso, os métodos históricos e biográficos, a etnografia no estudo dos fenômenos sociais e humanos. Na coleta dos dados, metodologicamente se dispõe a recolher e analisar dados: a entrevista qualitativa, estruturada ou semi- estruturada, a observação, a experiência pessoal, os métodos de análise de documentos, denotando uma pluralidade de técnicas que refletem a diversidade de objetos das ciências sociais e humanas e a necessidade de adaptar os métodos à sua especificidade. 0 rigor metodológico passa a ser um dos pilares da pesquisa social, acompanhado de estratégias a serem utilizadas no decorrer da coleta e análise dos dados. Entre elas, a prolongada presença em campo, descrições ricas do contexto e dos atores, diário de bordo, triangulação

2. Essas abordagens se resumem no pós-estruturalismo com R. Barthes, o neo-marxismo com Althusser, o descritivismo, com Geertz, as teorias culturais, com Turner, o desconstrutivismo com Derrida, a etnometodologia com Garfinkel. (ANADON, 2006, p. 10). 
(quali/quanti) etc, no sentido de permitir uma interpretação rica e detalhada do fenômeno estudado.

No campo da hospitalidade, igualmente, em que aos critérios científicos devem-se agregar os critérios éticos nas relações com "o outro", no caso pesquisadores/ pesquisados, assiste-se a estratégias múltiplas de abordagem dos fenômenos, recorrendo-se muito a estudos exploratórios, estudos aprofundados de casos únicos ou múltiplos, desde empresas hoteleiras e de serviços de toda ordem, até hospitais, comunidades, situações em que o recurso aos dados "qualitativos", como depoimentos, cartas, documentos, correspondências, etc., são um dos lados da questão. Em muitos casos, recorre-se igualmente aos dados quantitativos, à construção de índices e indicadores. Os estudos de levantamento do estado da arte em vários domínios, também têm sido utilizados, pela necessidade de levantar estudos em outros campos disciplinares. Além disso, constituem aspectos importantes nos estudos em hospitalidade, fenômenos de transposição de fronteiras como os movimentos migratórios e imigratórios, os movimentos de turistas entre regiões diferentes e seus impactos em comunidades, assim como pesquisas sobre o espaço urbano, as relações que se estabelecem no âmbito das cidades contemporâneas, no caso brasileiro, São Paulo e as demais metrópoles.

Há no momento atual também, um alargamento das fronteiras da pesquisa qualitativa, pela construção de historias de vida e biografias, suporte literário, as representações etc como fontes de dados, assim como a valorização da história do cotidiano, da hospitalidade urbana. A coleta de dados qualitativos, supõe uma grande diversidade de métodos de coleta de dados e procedimentos de campo, como enfatizam por exemplo, Bauer e Gaskell, (2002), que se dispõem segundo diferentes orientações teóricas na construção do que os autores chamam de corpus da pesquisa, que por sua vez, levam à discussão sobre a seleção dos sujeitos de pesquisa ou mesmo da "amostragem representativa" na pesquisa social.

\section{Metodologias qualitativas na eVolução do debate nas Ciências Sociais}

Como ressaltado anteriormente, no referido trabalho de 2010, "o aspecto diversificado da sociedade, foi tomando cada vez maior importância, à medida que sua natureza plural foi sendo constatada. [...] Com isso, tornou-se necessário que esta noção de plural fosse ressaltada e a sociedade estudada em seus diversos ângulos (QUEIROZ, 2001, p. 21) o que conduziu os campos disciplinares a se especializarem cada vez mais, e o que permite, a cada momento, que um se sobressaia sobre o outro, constituindo "formas de saber diferentes em seu objeto e, certamente, também em seus métodos, [...] colocando em evidencia a surpreendente desigualdade em seu desenvolvimento". (GUILLAUME, 1986, apud QUEIROZ, 2001).

[...] Novos paradigmas, gerados no âmbito da própria ciência como em outras áreas do conhecimento, vêm questionando pressupostos e procedimentos que até então orientavam a atividade científica e conferiam credibilidade aos seus resultados. A visão de uma ciência objetiva, neutra, a-histórica, capaz de formular leis gerais sobre o funcionamento da natureza, leis estas cujo valor de verdade seria ga- 
rantido pela aplicação criteriosa do método, já não se sustenta. Hoje, a maioria dos cientistas admite que o conhecimento nunca é inteiramente objetivo, que os valores do cientista podem interferir no seu trabalho, que os conhecimentos gerados pela ciência não são infalíveis e que mesmo os critérios para distinguir o que é e o que não é ciência, variam ao longo da história. (MAZZOTTI e GEWANDSNAJDER, 1998, p.109).

Se essas questões se colocavam para as ciências físicas e naturais, com maior ênfase se tornam importantes e cruciais para as ciências sociais e para o campo em construção, da hospitalidade. As pesquisas e os métodos ligados ao estudo dos fenômenos e fatos humanos não são passíveis de uma mensuração precisa e dessa forma, têm se adequado e se diferenciado da pesquisa dita "experimental", de base positivista que predominou nas ciências humanas depois do inicio do século XX e que postulava a necessidade de um conhecimento absoluto e de uma "verdade científica", baseados na quantificação e na medida, à maneira das ciências físicas e naturais. (ROBO, 1995, p. 1). Entretanto

[...] a complexidade da realidade social leva o pesquisador em ciências humanas a rever os princípios estabelecidos. Essa reavaliação incessante se acha presente desde os primórdios da sistematização da pesquisa social. [...] e as próprias pesquisas se encarregaram de demonstrar que o conhecimento dos temas humanos ou culturais envolve uma peculiar intimidade entre o pesquisador e seu objeto, não podendo portanto, reivindicar a mesma relação que existe nas pesquisas em ciências naturais. (BUENO, 2008, p. 7).

Na trajetória da evolução das ciências sociais, duas vertentes principais se instalam desde o inicio: uma que diz respeito à objetividade, pretendendo afastar todas as pré-noções para atingir um rigor metodológico, que tem em A. Comte e É. Durkheim seus principais representantes e outra, representada por uma sociologia "compreensiva", com M. Weber e a teoria da ação social, ressaltando a necessidade da compreensão do seu significado, que preconiza portanto, a compreensão dos fenômenos a partir do seu interior e de seus significados. A contribuição marxista (K. Marx e F. Engels), viria evidenciar o caráter contraditório da realidade social, exigindo uma análise profunda de suas estruturas e com isso, a necessidade de uma compreensão histórico-social dos fenômenos sociais, em oposição à noção de "invariabilidade" do social. Contemporaneamente, o debate em torno da objetividade/subjetividade evidencia uma flexibilização dos critérios de cientificidade, mantendo-se a discussão sobre a clareza e fundamentação do discurso científico. Considere-se que "a ciência tem por objetivo explicar os fenômenos, não apenas descrevê-los, e que esta característica, considerada essencial nas ciências naturais, é encontrada também nas ciências sociais". Realmente, "[...] a pesquisa em ciências sociais hoje se caracteriza por uma multiplicidade de abordagens, com pressupostos, metodologias e estilos narrativos diversos". (MAZZOTTI e GEWANDSZNAJDER, 1998, p.127).

Considere-se ainda, segundo os autores, que, a partir da década de 1960, e a chamada "crise dos paradigmas", intensificam-se os debates a partir das colocações de T. Kuhn (1970) sobre a objetividade e racionalidade da ciência e a retomada das críticas da Escola de Frankfurt (HORKEIMER, M. 1983), mostrando os 
aspectos ideológicos da ciência dominante que levaram à busca de modelos alternativos ao positivismo na criação do chamado "paradigma qualitativo", ou "novo paradigma". Esse processo, sobretudo a partir dos anos 1980, levaram entretanto, a uma falsa oposição qualitativo-quantitativo, mas sedimentaram, por outro lado, os caminhos da chamada "pesquisa qualitativa", que aprofunda a necessidade da compreensão dos fenômenos sociais segundo modelos alternativos ${ }^{3}$.

Pode-se dizer que as raízes antropológicas da pesquisa qualitativa ${ }^{4}$ encontram-se na evolução da antropologia evolucionista de finais do século XIX, e as preocupações com o entendimento comparativo e evolucionista das culturas e das dimensões biológicas e culturais, além da necessidade da própria conceituação de cultura, em autores como Tylor, Kröeber, F. Boas e Malinowski, L. Strauss, M. Mauss, e tantos outros mais recentes, que se preocuparam de inicio, com a necessidade de diferenciar a dimensão biológica da cultural nas sociedades humanas, no entendimento da vida social (LARAIA, 2001). A pesquisa baseada no acúmulo de dados etnográficos recolhidos a partir de notas de viagem de missionários, colonizadores, religiosos etc, no sentido de documentar as diferenças com o "outro", o "estranho", para uma pesquisa que preconiza a integração/ impregnação do pesquisador no meio estudado, marca um momento na evolução da pesquisa qualitativa que se estende ao início da Escola de Chicago, e representa uma evolução, caracterizada pelo cruzamento das disciplinas das ciências sociais, a chamada interdisciplinaridade.

Também de um ponto de vista sociológico e antropológico, a pesquisa qualitativa tem suas origens nos Estados Unidos, a partir de um contexto de crítica social oriunda das condições de vida de grande parte da população e desta forma, as questões como pobreza, imigração, marginalidade e criminalidade, passaram a ser os temas preferenciais dos pesquisadores e, nesse sentido, pode-se dizer que é à Escola de Chicago ${ }^{5}$ e seus trabalhos das décadas de 1920 e 30 a que se deve o chamado período tradicional de instauração da pesquisa qualitativa. Os pesquisadores produzem grande quantidade de trabalhos baseados na observação participante, na entrevista em profundidade, na história de vida e na análise de documentos pessoais, utilizados como metodologias no estudo de problemas que caracterizam a vida urbana, embasados no chamado interacionismo simbólico que postula que o comportamento humano não se compreende, senão em relação com as significações que as pessoas atribuem às coisas e às suas ações. (ANADON, 2006, p. 9,10). "Com os trabalhos da Escola de Chicago, a metodologia qualitativa ocupa um lugar importante na Sociologia americana, até

3. Nesse contexto, consolidam-se vertentes de análise baseadas no construtivismo social, no póspositivismo e na teoria crítica, cada uma das quais, fundamentada numa orientação filosófica, como a fenomenologia, a valorização da verificação da teoria e da objetividade, o empirismo, a análise rigorosa da argumentação e dos procedimentos de seleção, coleta e avaliação, buscando a consistência lógica entre eles, daí decorrendo metodologias e técnicas variadas de coleta e análise dos dados.

4. Para acompanhar a evolução e os desafios da pesquisa qualitativa, consistiram referências fundamentais as reflexões de: CHIZZOTTI, 2003; ROBO, 1995; ANADON, 2006.

5. A Escola de Chicago, como ficou conhecida pelas pesquisas do Departamento de Sociologia, criado em 1892, por W. Thomas e F. Znanieccki, foi responsável por uma vertente importante da pesquisa de caráter qualitativo, nas primeiras décadas do século XX, sobretudo com a entrada de Robert Park. Busca fundamentar uma metodologia para estudar "o outro", a partir das narrativas orais de história de vida cotidiana de pessoas comuns. (CHIZZOTTI, 2003). 
as décadas de 1940 e 50, em que os estudos baseados nos "hard data", evidenciam um novo crescimento nas ciências humanas e sociais". (ANADON, 2006, p. $10)$.

No período seguinte, que vai do final da Segunda Guerra Mundial aos anos 80, consolida-se a utilização de perspectivas teóricas das ciências sociais, misturadas à Semiótica e à Hermenêutica, que acabam por causar grande confusão nas práticas de pesquisa, provocando o aparecimento de novas abordagens teóricas como o pos- estruturalismo (Barthes), o neo-marxismo (Althusser), o descritivismo (Geertz), o desconstrutivismo (Derrida), a etnometodologia (Garfinkel), etc, período em que se busca uma sistematização de diferentes técnicas de coleta e análise de dados qualitativos (entrevistas, observação, análise de documentos, etc). Valorizam-se as interpretações que os atores sociais têm da realidade, o que entretanto, é entendido também como uma dificuldade na validação dos dados dos modelos interacionistas e construtivistas de pesquisa, da fenomenologia, da sociologia crítica, etc e das formas de pesquisa, como os estudos de campo, a pesquisa in loco, natural, a etnografia, grounded theory, etc. (ANADÓN, 2006, p. 10).

A maneira de conceber a pesquisa qualitativa referindo-a apenas às suas técnicas particulares, é muito restritiva pois a pesquisa qualitativa deve estar associada às posturas teóricas e epistemológicas que privilegiem de uma forma ou de outra, a experiência e os pontos de vista dos atores sociais. Assim, a pesquisa deve se caracterizar mais pela postura adotada teoricamente, do que pelo tipo de dado produzido. (ANADON, 2006).

Realmente, a particularidade da pesquisa qualitativa pode ser resumida pela relação que estabelece e produz, entre uma postura epistemológica definida, do pesquisador, e a natureza dos dados.

"Muitos esforços foram despendidos na tentativa de justapor pesquisa quantitativa e qualitativa, como paradigmas competitivos de pesquisa social [...]", (BAUER e GASKELL, 2002, p. 23). Entretanto, a própria mensuração dos fatos sociais, depende da construção de categorias de interpretação (BAUER e GASKELL, 2002, p. 24) e, entende-se com os autores que, os métodos quantitativos ou qualitativos, são mais do que diferenças entre estratégias de pesquisa ou procedimentos de coleta de dados, mas representam fundamentalmente, diferentes referenciais epistemológicos para teorizar a natureza do conhecimento, a realidade social e os procedimentos para compreende-los. (p. 29).

Desta forma, retomando a reflexão de Camargo, (2015) e Caillé (1998), sobre a importância do novo paradigma do dom e da hospitalidade para a pesquisa social, a hospitalidade é mais do que um campo de estudo previamente delimitado, como ocorre nas ciências puras e aplicadas, mas apresenta-se como um novo paradigma para o estudo do relacionamento humano, uma nova perspectiva, um novo olhar. (Camargo, 2015, p. 48). Não se deve esquecer que além da ênfase na contribuição de Marcel Mauss no Ensaio sobre o dom, os intelectuais franceses participantes do Movimento antiutilitarista em Ciências Sociais, têm insistido na contribuição de Karl Polanyi, na sua obra de 1944, A grande transformação, as origens da nossa época, em tradução brasileira de 2000, em que o autor realiza profunda crítica da visão economicista clássica, liberal que atribui às transformações econômicas, o motor da mudança social. Nessa critica, se resumem 
basicamente, os fundamentos do movimento antiutilitarista em ciências sociais. Realmente, diz Polanyi, (2000, p. 56):

No coração da Revolução Industrial do século XVIII, ocorreu um progresso miraculoso nos instrumentos de produção, o qual se fez acompanhar de uma catastrófica desarticulação nas vidas das pessoas comuns. [...] Qual foi o mecanismo por cujo intermédio foi destruído o antigo tecido social e tentada, sem sucesso, uma nova integração homem-natureza? A filosofia liberal jamais falhou tanto como na compreensão do problema da mudança [...] na aceitação mística das consequências sociais do progresso econômico.

Assim, "a convergência entre Karl Polanyi e Marcel Mauss é tocante, uma vez que suas analises econômicas encontram sua origem em uma mesma crítica reducionista, que explica a ação econômica apenas pelo interesse material individual". LAVILLE, 2004, p. 43).

De fato, ao contrário do que se espera pelo entendimento do funcionamento do mercado como integrador, surgem pelo menos dois problemas extremamente graves: "a desagregação dos princípios organizadores da solidariedade social e o fracasso da concepção tradicional dos direitos sociais que ofereça um quadro satisfatório para explicar a exclusão social" (INNERARITY, 2001, p. 315).

Além disso, ainda segundo o autor, enquanto no pós-segunda guerra mundial, o núcleo da questão social estava na redistribuição, "o principal acontecimento de nossas economias é a exclusão de grupos inteiros do mercado de trabalho" (p. 314), o que leva a uma nova justificativa da intervenção pública na redução das desigualdades, que no entanto, não assegura a coesão social. Sem discutir a qualidade dessa intervenção, realmente, o que está em jogo no centro da questão social, são os princípios organizadores da solidariedade (p.316) que os enfoques exclusivamente econômicos tendem a ignorar.

Assim, como enfatiza Innerarity (2001), a categoria da hospitalidade pode servir para articular uma teoria moral, em virtude de sua universalidade cultural e riqueza de suas significações. Com a categoria da hospitalidade, diz o autor, "pretendo dar fundamento conceitual aos acontecimentos que escapam à previsão e ante os quais, se mede nossa verdadeira estrutura moral" (p. 18), não se tratando aqui de uma abdicação da razão, mas tratando-se a ética da hospitalidade, como a aprendizagem do trato produtivo com a alteridade. "A competência ética fundamental consiste na abertura ao outro e aos outros, em estar acessível aos apelos do mundo, atento ao diferente de si mesmo" (p. 19). Assim, a hospitalidade num mundo atravessado pelos interesses particulares e pelo individualismo, caminha em direção diferente. "Interessam-lhe a proximidade e o encontro, e este é talvez, o seu principal significado, face às lógicas da globalização e do individualismo" (CAMARGO, 2015, p.44).

\section{CONSIDERAÇões finais}

Objetivou-se nesse espaço, evidenciar a importância do social face ao econômico estritamente considerado como o motor das mudanças sociais, e trabalhar com as relações entre a pesquisa qualitativa e o campo da hospitalidade, no sen- 
tido, também, de evidenciar suas relações, no contexto das profundas fraturas e contradições que caracterizam a contemporaneidade.

A dimensão, a categoria ou o campo da hospitalidade, permitem a crítica à visão estritamente economicista das mudanças sociais e suas consequências para a vida em sociedade. Face ao esgarçamento do tecido social e à progressão dos problemas sociais na contemporaneidade, a pesquisa em hospitalidade dá continuidade à tradição da pesquisa social qualitativa, que visa resgatar o sentido e a manutenção da vida em sociedade, enfatizando a importância dos vínculos sociais. Nesse sentido, a pesquisa dita qualitativa, encontra suporte nas discussões atuais sobre a hospitalidade, como se procurou evidenciar.

\section{REFERÊNCIAS}

ANADON, M. (2006). La recherche dite "qualitative": de La dinamique de son évolution aux acquis indéniables et aux questionnements présents. Recherches Qualitatives, V. 26, (1), 5-31. Association pour La recherche qualitative (ARQ) e Conseil de recherche em Sciences humaines Du Canada (CRSHC), Université Du Quebec à Trois Rivières (UQTR).

BAPTISTA, Isabel. (2002). Lugares de hospitalidade. In: DIAS, Célia M. M. (Org.) Hospitalidade: reflexões e perspectivas, São Paulo: Manole, 2002.

BAUER, M. W.; GASKELL, G. (2002). Pesquisa qualitativa com texto, imagem e som. Um manual prático. Petrópolis: Editora Vozes.

BUENO, M. S. (2008) (Org). Hospitalidade no jogo das relações sociais. São Paulo: Editora Vieira.

CAILLÉ, A. (1998). Nem holismo nem individualismo metodológicos: Marcel Mauss e o paradigma da dádiva. Revista Brasileira de Ciências Sociais. V. 13, no. 38 (1-15). São Paulo: Outubro de 1998.

CAMARGO, L. O. L. (2003). Os domínios da hospitalidade. In: DENCKER, A. de F. M. e BUENO, M. S. (Orgs). Hospitalidade: cenários e oportunidades. São Paulo: Thomson.

ro Especial.

(2015). Os interstícios da Hospitalidade. Revista Hospitalidade. Núme-

CALHOUN, C. (2014). 0 papel das Ciências Sociais em um mundo em mudança acelerada. Revista FAPESP, agosto. São Paulo: Fundação de Amparo à Pesquisa do Estado de São Paulo.

CHIZZOTTI, A. (2003) A pesquisa qualitativa em Ciências Humanas e Sociais. Evolução e desafios.. Revista Portuguesa de Educação. Ano 16.V.16, 002, Universidade do Minho. Braga, Portugal, (221-236).

GOTMAN, A.(1997) La question de l'hospitalité aujourd'hui. Communications. V. 65, no. 65, no. especial: Hospitalité, (5-19).

HORKEIMER, M. (1983). (Org.) Teoria tradicional e teoria crítica. In: CIVITA. Textos escolhidos. V, São Paulo: Abril Cultural.

INNERARITY, D. (2001). Ética de La Hospitalidad. Barcelona: Ediciones Peninsula.

KHUN, T. (1970). The structure of scientific revolutions. Chicago: University of Chicago Press,

LARAIA, R. B. (2001). Cultura, um conceito antropológico. 14a‥ Edição. Rio de Janeiro: Jorge Zahar Editores. 
LASHLEY, C. e MORRISON, A. (2004). Em busca da hospitalidade: perspectivas de um mundo globalizado. São Paulo: Editora Manole.

LASHLEY, C. (2015) Hospitalidade e hospitabilidade. Revista Hospitalidade. V. XII, no. especial. Maio, 2015, (70-92).

LAVILLE, J. L. (2004). Com Mauss e Polanyi, rumo a uma teoria da economia plural. In:

LUGOSI, P. (2008). Hospitality spaces, hospitable moments: consumer encounters and affective experiences in commercial settings. Dorset: Peter Lugosi School of Services Management, Bournemouth University, Talbot Campus, Poole, BH12 5BB, UK.

MARTINS, PH e NUNES, B.F. (2004). A nova ordem social: perspectivas da solidariedade contemporânea. Brasilia: Paralelo 15 Edições.7

MARTINS, P. H. e NUNES, B.F. (2004) (Orgs). A nova ordem social. Perspectivas da solidariedade contemporânea. Brasilia: Paralelo 15 Edições,

MARTINS, P.H. (2007) Apresentação ao Jornal do M.A.U.S.S., Recife: Universidade Federal de Pernambuco.

(2005). A Sociologia de Marcel Mauss: dádiva, simbolismo e associação. Revista Crítica de Ciências Sociais, 73, Dezembro. (45-66).

MAUSS, M.. (1925). Essai sur Le Don. Forme et raison d'échange dans lês societés archaïques. Année Sociologique, 2d. série T.1.

(1974) Antropologia e Sociologia. São Paulo: EPU/EDUSP.

(2001) Ensaio sobre a dádiva. Edições 70, 2001.

MAZZOTTI, A. J. A. e GEWANDSNAJDER, F. O método nas Ciências Naturais e Sociais. Pesquisa Quantitativa e Qualitativa. São Paulo: Pioneira, 1998.

MONTANDON, A. (2003) Hospitalidade ontem e hoje. In: DENCKER, A.F. M. e BUENO, M. S. (Orgs). Hospitalidade: cenários e oportunidades. São Paulo: Thomson.

POLANYI, Karl. (2000). A grande transformação: as origens de nossa época. Tradução Fanny Wrabel, $2^{\underline{a}}$ edição. Rio de Janeiro: Editora Campus.

QUEIROZ, M.I. P. de. (2001). Problemas na proposição de pesquisas em Ciências Sociais. In: LANG, A. Beatriz da Sila Gordo (Org.). Textos CERU. Desafios da pesquisa em Ciências Sociais, no 8, Série 2. São Paulo: Humanitas. FFLCH/USP.

ROBO, Patrick. (1995). A propôs de recherche et de methodes qualitatives. Ecrits Perso. Site PR/Méthodes qualitatives.doc. (Documento elaborado a partir de: POURTOIS J-P. (1988). et DESMET H. Epistémologie et instrumentation en Sciences Humaines. Paris: Mardaga, MUCCHIELI A., Paris: PUF, Coleção Qui sais jê, no. 2591, 1991).

SALLES, . R. R.; BUENO, M.S.; BASTOS, S. R. (2010). Desafios da pesquisa em hospitalidade. Revista Hospitalidade. V. VII, Número 1, Julho de 2010.

Recebido em: 10/08/2016

Aprovado em: 25/09/2017

\section{Contribuição}

MARIA do Rosário Rolfsen SAlles: Autoria Única. 